\title{
Child Healthcare in Nepal: Progress and Direction
}

\author{
Radeeb Akhtar \\ New York Medical College, Valhalla, U.S.A \\ *Corresponding author: radeeb.akhtar@gmail.com \\ Received January 31, 2015; Revised March 15, 2015; Accepted March 22, 2015
}

\begin{abstract}
Health policy changes in Nepal displayed struggles against a poor political, geographical, and economic setting; Millennium Development Goal \#4 demanded improved infant and child mortality, as well as adequate measles vaccine coverage by the year 2015. Research in this report presents progress and direction of child health care policy across more than a decade of time in attempts of attaining MDG \#4 and general child health care advancements. Subsequent observations and suggestions were delineated and offered. Progress since the 1990's up to 2012 was analyzed by review of serial national survey and report data. Trends and variations between regions were mostly analyzed amongst various child health care determinants. Results indicated many improved factors; Nepal will likely achieve MDG regarding child under-5 mortality, but may not achieve measles vaccine coverage or infant mortality goals. Furthermore, severe regional disparities were evident within Nepal, particularly in the Mid and Far-Western regions. A call for integrated community-based primary health care (CB-PHC) for infants and children became an ultimate ideal. A comprehensive, multidisciplinary, and community based primary care delivery service would address many of the deficits identified as well as reach rural and remote areas that still suffered. Risk and data-based resource allocation promise improved utilization, but also demands more frequent and better data reporting. Coordinated, multi-sector health policy initiatives have been underway; this demonstrated a strong direction in improving child health care from urban to every village.
\end{abstract}

Keywords: Nepal, child healthcare, health policy, infant mortality, child mortality, child immunization, millennium development goal, community medicine, community based integrated healthcare

Cite This Article: Radeeb Akhtar, "Child Healthcare in Nepal: Progress and Direction.” American Journal of Public Health Research, vol. 3, no. 2 (2015): 74-80. doi: 10.12691/ajphr-3-2-7.

\section{Introduction}

The United Nations' Millennium Development Goal (MDG) \#4: Reduce Child Mortality aims to implement health policy to improve child healthcare. This research shows Nepal's implementation for advancing child healthcare and further provides suggestions for health policy makers in Nepal. MDGs were proposed by the UN to implement and finance initiatives all over the world in efforts to address the most important global health and human rights concerns. MDG \#4 declared that by 2015, various health indicators would be a reduced to certain rates. Target rates include three primary components: 1 . Infant Mortality of 36 per 1,000 live births, 2. Child Under-5 Mortality of 54 per 1,000 live births [4]. Proportion of one-year-old children immunized against measles for $>90 \%[2,14]$.

Initiatives to improve child healthcare and overall mortality includes immunization coverage, addressing malnutrition, and acute illness prevention and treatment (specifically respiratory illness and diarrheal disease). Figure 1 demonstrates the geographic variation based on municipality regions as well as terrain regarding child growth stunting in 2011. Nepal is divided into 5 municipality regions named Eastern, Central, Western, Mid-Western, and Far-Western. Each region has unique characteristics and variable terrain. Most prominently delineated in Nepal is the three ecological belts, as seen in color on Figure 1: Terai or subtropical, Hills, and Mountain. Child growth stunting represent malnutrition concerns, which are apparently highly variable in 2011, and seem to correlate with ecological background more so than municipality [11].

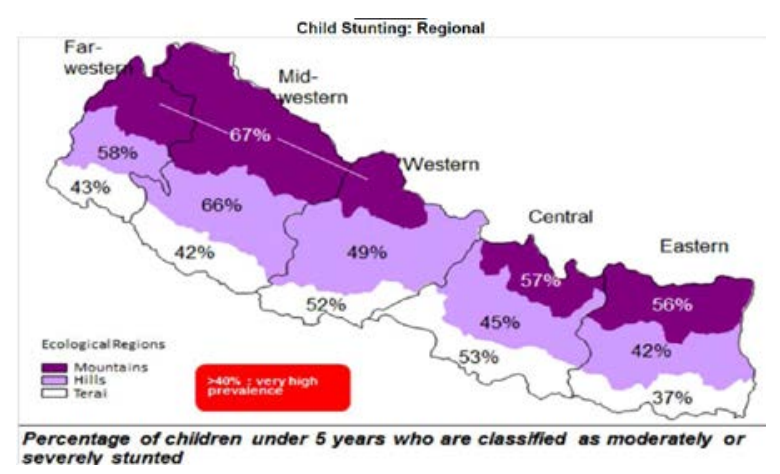

Figure 1. Child growth stunting based on region (labeled) and terrain (shaded) in Nepal, 2011 [11]

Health policy initiatives began long before the MDGs; child mortality, especially in geographically disparate communities since the National Healthcare Policy in 1991, then via reform many years later. The idea of healthcare as a fundamental right only echoed the need to increase access to care [10]. Policy initiatives included expansion 
the National Immunization Program then the Multi Year Plan of Action, the National Nutrition Program, and Community Based Integrated Management of Childhood Illness to address immunization coverage, malnutrition, and acute illnesses respectively. These methods utilized outreach clinics, mobile vans, and female community health workers $[2,3,4]$.

Hoping to develop the basic determinants of child health, the Ministry of Health often had dealt with the challenges of healthcare infrastructure, finances, and the burden of national poverty. Large contributors that have been shaping child healthcare in Nepal include firstly the health care reform in 1991 which called for increasing health standards and focused on reducing infant and child mortality. Changes made during this reform include decentralization of the prior health care system. A new constitution in 2007 declared basic health care as a fundamental right for every citizen, specifically recognizing the needs in rural communities, women, and children [12]. The National Immunization Program or NIP and the Multiyear Plan of Action or MYPA were government run and highly prioritized national vaccination campains. The community based integrated management of childhood illness or CB-IMCI was launched in 1997 and progressively expanded the program amongst districts. The National Nutrition Program, The School Health and Nutrition Strategy, and Scale-Up Nutrition (SUN) represent the course of action health policy has been acting against malnutrition and poverty $[2,3,4,12]$.

\section{Methodology}

Health policies implemented and health determinants reflecting was analyzed adjacently. Analyses of data included multiple annual reports (years 2006 to 2012) published by the Department of Health Services (covering 2004-2012), Government of Nepal Ministry of Health and Population, and child health surveys conducted annually (1996-2010). Serial analysis provided an extended temporal demonstration of health policy performance as well as response from the population for more than ten years.

Health indicator data collected and reported amongst mostly all annual reports. This data was trended and interpreted.

Health Indicators:

- Child Under 5 Mortality

- Infant Mortality

- Vaccine Coverage

- Vaccine Drop-Out Rate

- Mortality due to Vaccine Preventable Disease

- Growth Monitoring Visits

- Malnutrition

- Nutrition Supplementation Distribution

- Incidence of Acute Illness

- Treatment of Acute Illness

Interventions mentioned per annual reports employed by public health care systems were analyzed adjacently to statistics. Some of these include major health policy and system changes (decentralization), NIP, MYPA, The National Nutrition Program, CB-IMCI, etc.

\section{Results}

Reports demonstrated a steady decline in mortality as seen in Figure 2, likely largely due to early recognition and treatment of acute illness. Much of efforts working against acute illness involve the CB-IMCI. Health facilities and outposts, mobile vans, and female village health volunteers were primary means of data gathering and delivery of services, CB-IMCI. The means of delivery proved to be effective in not only the prevention of severe acute illness, but also administration of immunizations specifically in the setting of nationally employed immunization campaigns. Please refer to Figures 5 and 6 to view trends for Measles coverage and Acute Illness: Diarrhea respectively. These results are delineated by side by side analysis of national statistics reported. Disparities were observed between terrain and region municipalities, though the gaps in care seems to narrow throughout the years as seen by Figure 3 and Figure 4.

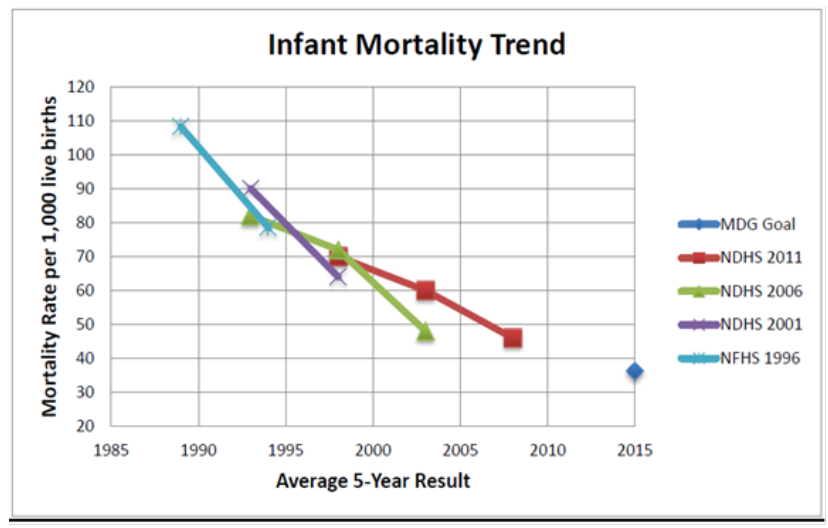

Figure 2. Infant Mortality: Multi-Year Progression.

Infant mortality dropped from a rate of 108 deaths per 1,000 live births in 1990 down to 46 in 2011. Last two reports do not show a decrease in infant mortality, and the latest reported national rate was not at MDG goal. See Figure 2 for staggered reports in a multi-year progression. Neonates to two years prove to be the most vulnerable age.

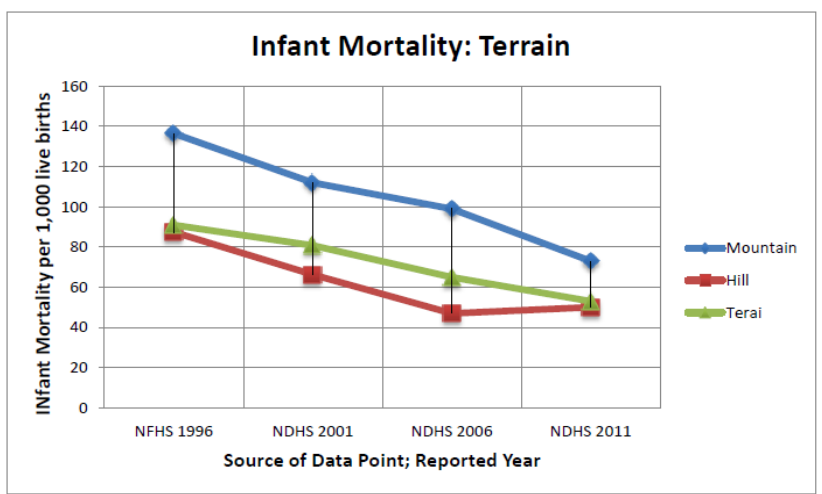

Figure 3. Infant Mortality: Multi-Year Progression; Terrain.

Large regional differences were demonstrated, with the Mid and Far Western regions harboring worst rates. Furthermore, urban versus rural also presented disparity; infant mortality was $43 \%$ lower in urban settings compared to rural [10]. Data showed that in 2011, the urban infant mortality did meet MDG goal, while rural infant mortality remained markedly higher though 
decreasing with a narrowing disparity gap. Terrain also played a large role in terms of disparities in mortality and morbidity as seen in ecologically stratified data; mountainous terrain along the northern belt carried a heavier mortality burden (Figure 3), malnutrition rates, as compared to southern, hill and subtropical terrain.

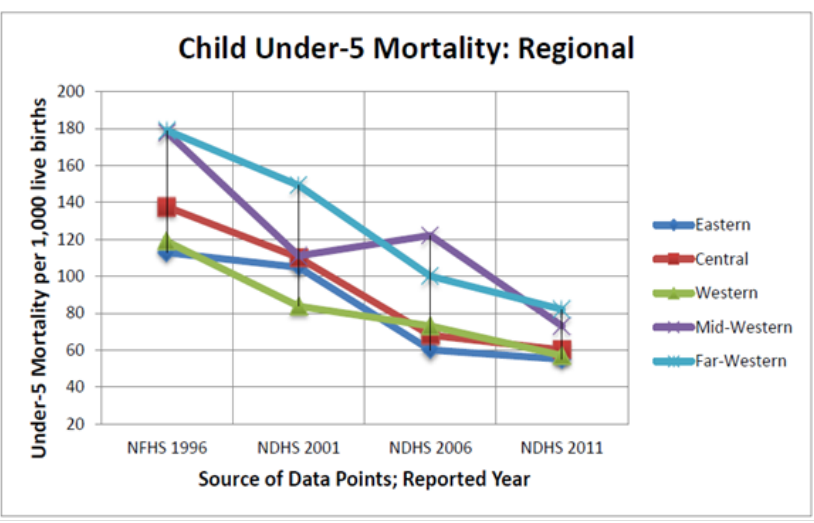

Figure 4. Child Mortality: Multi-Year Progression; Regional

Child under-5 mortality was reported to be 195.6 per 1,000 live births in the 1980's to the latest report of 54 (2005-2010) which is below the MDG goal. Mountainous regions suffered worse child mortality than hill or Terai terrain. Throughout the years, the disparities between terrain have been narrowing. The slope in the mountain under-5 mortality trend was steeper compared to other terrains. Differences between regions were also noted Mid and Far Western regions initially demonstrated the worst under-5 mortality rates which have remarkably been improving. While the national Under-5 mortality rate was most recently below goal, the far west region of Nepal had a mortality rate of 82, very far from the MDG goal.

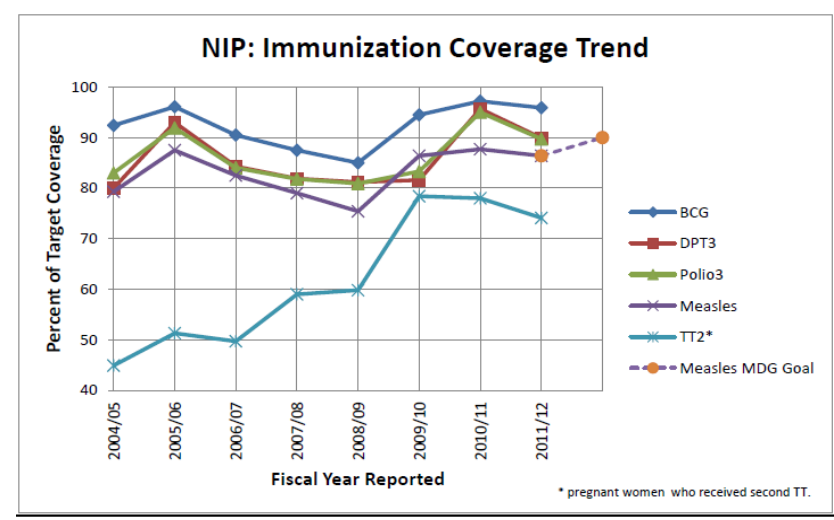

Figure 5. National Immunization Coverage: Multi-Year Progression

Immunization coverage for Measles trended two demonstrable peaks as seen in Figure 5. All immunization coverage also demonstrated this. The national Measles coverage went from $80 \%$ to $88 \%$ of infants receiving the Measles vaccine before the age of one year. Tetanus vaccination markedly increased throughout the years, though recently declined. The more recent 2-3 year reports indicate a decline in all immunization coverage, including Measles. The latest report indicated that Nepal was not within MDG goal (>90\%).

Measles coverage throughout the years remained worst in the Western region, as seen in Figure 6. Interestingly, the Mid and Far Western regions had the best measles coverage from latest reports. Specifically, the Far-Western region trend went from the worst Measles coverage in 2004 to one of the highest coverage rates and above the MDG goal in 2011.

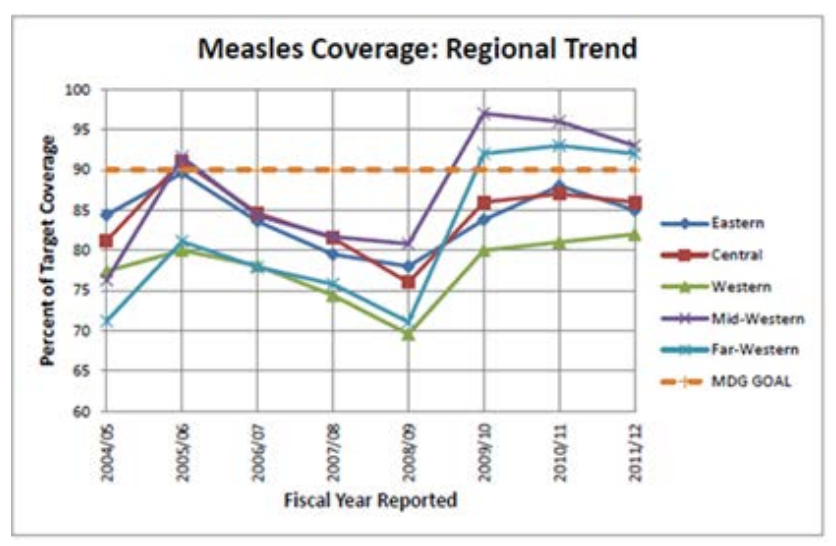

Figure 6. Measles Coverage: Multi-Year Progression; Regional

Standard vaccine schedule has changed somewhat throughout the years in response to disease prevalence and community need and resource allocation. Changes noted include dropping Tetanus from NIP to school based coverage and uptake of vaccination against Japanese Encephalitis (JE), as well as newer H. Influenza type B and Measles-Rubella combination vaccines.

Vaccine drop-out rates were analyzed as well (not shown here). Drop-out rates for DPT3 (diphtheriapertussis-tetanus vaccine - third dose) generally improved in more recent years, but in light of worsening allimmunization coverage. Mid and Far Western regions harbor the worst drop-out rates. Furthermore, BCGMeasles drop-out rates were also measured, showing positive in all regions (persistently worst in the Central Region).

Malnutrition and growth stunting was tracked according to under-3 then under-5 growth visits. There was an expansion in data collection according to said age groups. Growth tracking increased gradually on national parameters. Regionally, growth visits excelled in the MidWestern and dropped in the Far-Western regions. Comparing under-3 to under-5 data, there were many 3-5 year old that were not being tracked (not shown here). Number of visits from 2008 at about 2 visits increased to about 2.6 visits most recently reported.

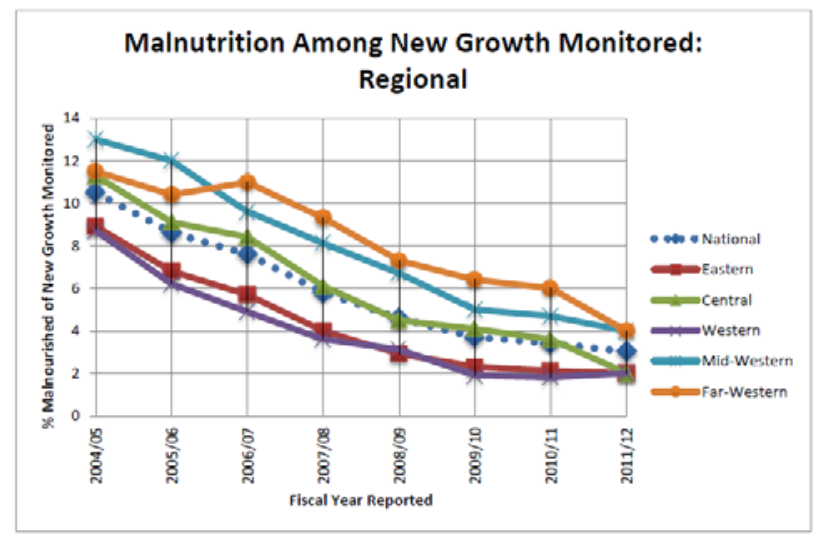

Figure 7. Malnutrition: Multi-Year Progression; Regional

Malnutrition was reported from those growth monitoring visits. In general, malnutrition has been declining amongst children nationwide; from $>10 \%$ of 
children growth monitored to about 3-4, in light of increased growth monitoring. Also noted were the regional disparities as seen in Figure 7 . These disparities seemed to have narrowed somewhat, especially in the latest report. Mid and Far Western Regions harbored the worst rates of malnutrition persistently throughout the years. There was not enough data on malnutrition otherwise or micronutrient deficiencies across several years.

Data collection of both diarrhea and acute respiratory illness incidence improved with the elaboration of surveillance; thus, rates of incidence of both acute disease processes show marked increase at 2006 to 2009. There was an indirect relationship between incidence of diarrhea and percentage of severe dehydration year after year. Latest reports in 2011/12 indicated a less than 0.5\% of total diarrhea cases suffered from severe dehydration, as opposed to a national rate of $2 \%$ of diarrhea resulting in severe dehydration as seen in 2004. Mortality due to diarrhea varies depending on the year, but the burden was generally seen to be declining from rates of over 200 in 2004 to more recently less than 50 in 2011. The worst rates of incidence, mortality, and severe cases were found to be in the Mid and Far Western regions.

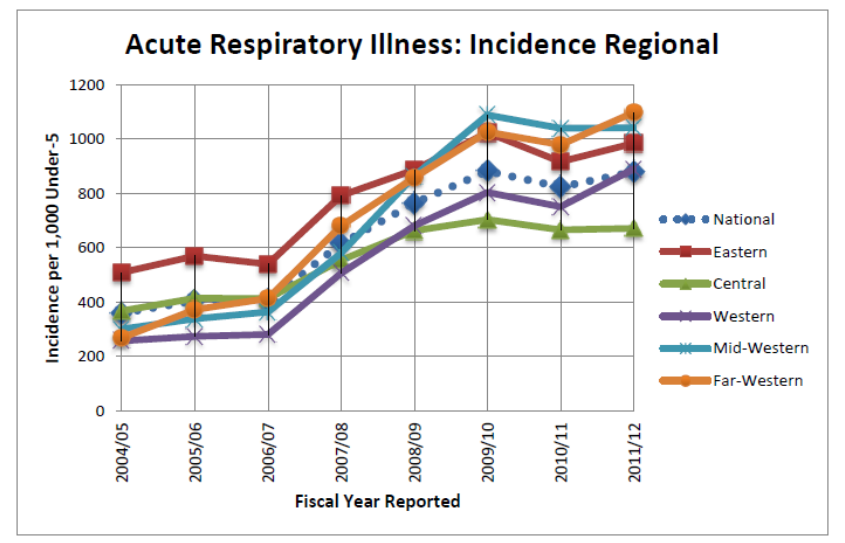

Figure 8. ARI: Regional Incidence - Multi-Year Progression

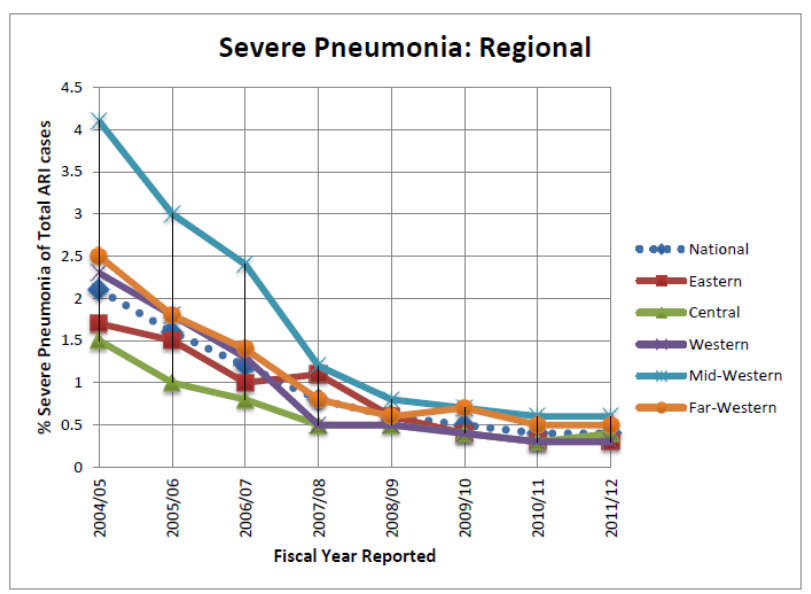

Figure 9. Severe Pneumonia: Multi-Year Progression; Regional

Acute respiratory illnesses held a higher mortality burden than diarrhea and related dehydration according to data (not shown). Incidence increased due to improved surveillance systems in 2006 (as seen in Figure 8), which seems to plateau starting 2009/10. Alongside increased incidence was also a decrease in severe pneumonia (Figure 9) and increase in treatment with antibiotics (not shown here). Disparities apparent in Figure 9, especially high rates of severe pneumonia in the Mid-Western region have seemed to level off from 2010. Interestingly, mortality burden has not remarkably decreased much since 2004 despite significant reduction in percentage of severe pneumonia cases. Regions with higher ARI mortality also some years tended to treat with home remedies rather than antibiotics.

\section{Discussion}

According to trends, Nepal will likely reach MDG \#4 targets specified child under-5 mortality, though may not reach MDG regarding infant mortality or immunization coverage of measles by 2015 [2,3,4,10].

There has been significant improvement in mortality rates which likely reflect the progressive overhaul of the health care systems and more effective delivery of services in recognized areas and communities of need. Community based integrated care seems to be the means of this effective delivery, with also in a setting of likely somewhat improved financial and political support in child health care, including the MDGs themselves [11].

Gaps in topographic and regional health care were apparent in said research. Specifically, mountainous and rural communities suffered the most mortality and morbidity burdens as seen in all data. Typically, the Mid and Far Western regions carried heavier mortality burdens as well as suffers the most poverty. Latest reports demonstrate a rural infant mortality of 64 deaths in 1,000 live births for 2012 compared to the lower urban rate which, if looked at alone, had achieved MDG \#4 goal [4].

Strides in the past decade has proven to narrow these gaps in health care between region and topography, as seen in data stratified by region and between the three topographic belts. It became clear that addressing vulnerable and minority populations in Nepal is key in improving child health care overall. This gap-closing phenomenon was likely due to community based interventions and recognition of need in these disparate communities. Health care disparities in mountainous regions remain a challenge in Nepal. These areas have been associated with reduced access to care, less tools and health care resources, and poorer health services (facilities and personnel). These areas also harbor concerns in land cultivation and agriculture which was reflected in rates of poverty, growth stunting, and malnutrition. The concerns in these underdeveloped communities are multifactorial $[8,13]$.

Immunization expansion has played a large role in reduction of mortality and morbidity via reducing vaccine preventable diseases. The NIP and MYPA were high priority programs in the government's agenda, and both resulted in the double-peak in coverage observed across the years. It was clear that these programs had significant impact, but remained effective for only a few years then faded. Most recently was seen the decline in all immunizations and measles coverage, failing to make the $>90 \%$ MDG goal as of 2012. Furthermore, it has been demonstrated the beneficial impact and efficacy of prioritization of immunization programs and delivery of these services. Topographically difficult regions such as the Mid and Far West has shown improved immunization 
coverage, demonstrating effective implementation in outreach to these areas that otherwise harbor high acute illness mortality and malnutrition [4,7].

Drop-out in immunizations for DPT3 improved throughout the years implying sophistication of vaccine delivery and cultural acceptance of vaccines and boosters. Mid and Far Western regions have the worst follow up, and call for increased mobile outreach and promotion of utilization of services. Reports indicated that the goal for four mobile visits per year was not always attained. The regional and inter-vaccine differences in distribution of routine immunizations suggest that there has been a lack in standardization of childhood vaccines. If standardized, rates of distribution and coverage should be similar in DPT1, Measles, BCG, etc. BCG-Measles drop-out >10\% throughout the years represents the lack of this. Considering this drop-out, BCG is typically easier to administer since it was given at first medical contact with infant, while Measles at an older age requiring later follow up [4].

Considering that immunization coverage improves with increased financial support and political backing, the more recent reduced rates were likely due to budget and reduced efforts. Measles coverage in the Far-Western region impressively changed from lowest (2004) to best covered region and above the MDG goal (2012). This demonstrated the potential power of allocated health policy interventions. Meanwhile, the Western region has persistently held worst rates of Measles vaccine coverage, possibly due to resource allocation; though, in the setting of lower malnutrition, poverty, and acute illness rates in comparison to other regions, it was unclear why this region suffered the worst Measles vaccine coverage rates throughout the years $[2,5,7,10]$.

Monitoring of vaccine drop-out rates and vaccine preventable disease incidence can allow for proper policy implementation and targeting toward vulnerable communities. This would allow efficient resource allocation in the setting of a resource poor nation. Recognition of Japanese Encephalitis as an increasingly prevalent and untreatable disease prompted the Ministry of Health to employ JE vaccine coverage across more affected districts. A recent report indicated a vaccine coverage of $88 \%$ of national target [4]. The NIP and MYPA had recognized behavioral change as means of increasing utilization of services: some employed interventions include National Immunization Days, some suggestions include incentivization, political advocacy, budget changes, etc.

One third of child mortality has been related to malnutrition and often make an otherwise self-limiting illness fatal. Growth stunting has always been impressively prevalent in Nepal, even in recent reports (as seen in Figure 1). Malnutrition and stunting reflect a more complex issue in Nepal - related to poverty, agriculture, and economics [11,13]. Growth monitoring expanded in surveillance, and increased slightly from 2 to 2.6 growth visits since 2004 to latest report 2012. This measurement alone was determined to be unsatisfactory since growth trending would require multiple visits to trend growth curves. Reports do not mention how growth was tracked at home or at school. Nonetheless, malnutrition did indeed decline from 10\% (2006) to 3\% (2012) nationally, worst in the Mid and Far Western regions. Malnutrition distribution was related to level of poverty. Unfortunately, malnutrition has likely been highly underreported in the light of meager growth monitoring nationally. Child mortality due to malnutrition was never measured, and has likely been a large contributor to morbidity and mortality. Malnutrition was also seen to be associated with low caste status, specific sub-cultures, and in accordance with topography [13].

Interventions of micronutrient distribution such as iodization, vitamin-A, and iron-folate have been underway and studied in more recent annual reports with promising results [1]. Nutrition deficiency conditions were not monitored and would certainly be beneficial in determining need of distribution and allocation of supplies. Maternal anemia in pregnancy was reported in one study to be effectively treated with iron-folate, demonstrating a $31 \%$ decline in early infant mortality and low-birth weight. To note, hemoglobin levels were not measured $[1,8]$.

The School Health and Nutrition Strategy initiated in 2006 and was a public effort to combat malnutrition and growth stunting nationally. The project included physical checkups, deworming, and reviewing nutritional behaviors. The impact of this program was uncertain, targeted only schooled children, and failed to address early infant malnutrition, breast feeding concerns / education. ScaleUp Nutrition (SUN) was the newest initiative in Nepal, started in 2010. This new project focuses on a multsectoral approach to addressing poverty and malnutrition. SUN describes efforts to be either nutrition specific and nutrition sensitive. Nutrition specific efforts were those within the health sector, on newborns within the "window of growth failure" which occurs at early infancy, and micronutrient supplementation. Nutrition sensitive efforts involved non-health sector participation, including food security, agriculture, education, sanitation, engineering, etc. SUN brought forth the concept of broad partnerships and political support. Outcomes of SUN presented by the National Planning Commission reported improvement in practices that promote nutritional services, multi-sector coordination at national and local levels, and expansion of capacity and utilization of basic services. A Nutrition Assessment and Gap Analysis in 2009 recommended maintenance of vitamin-A supplementation, deworming, treatment for diarrhea, salt iodization with expansion of micronutrition powders for younger children, infant feeding, and flour fortification [8,10,11,13].

Two major child killers in Nepal include acute respiratory and diarrheal diseases. Detection of disease incidence have enhanced substantially since 2006, with more reliable data as of 2009. The transition to adequate data collection was due to improvements of the Health Management Information Systems and their collaboration with CB-IMCI. Data reports trended revealed some large mortality burden at certain years which may be representative of natural disaster. The Far Western region suffered a high mortality in several annual reports, such as 2009/10. Oddly, that year also reported minimal use of IV fluids as treatmet. This regional disparity may be due to inappropriate medical response, underreporting, poorcommunity based care. The use of oral rehydration therapy and zinc became a form of treatment in $90 \%$ of cases, demonstrating marked improvement across all regions. In $2011 / 12$, less than $0.5 \%$ of total diarrhea cases suffered severe dehydration. 
ARI and pneumonia show a preferential incidence in Eastern, Far and Mid Western regions with every child at some point below 5 years afflicted. Mountainous topography make up most of the terrain in these three regions, though no terrain based data was reported for incidence of ARI or pneumonia. There was a notable shifting mortality burden demonstrated with mortality data between regions. This shifting may be due to illness epidemics (such as a geographic spread of a more virulent strain of flu), reallocation of resources, or natural disasters. The Mid-Western region suffered a higher mortality consistently across years. This region also has increased incidence and a higher percentage of cases were categorized as severe pneumonia. Furthermore, there was a national shifting of increased use of antibiotics for treatment. Both the Mid-Western and Eastern regions (higher ARI mortality) had increased utilization of antibiotic treatment. There was less use of antibiotic treatment in the Far-Western region compared to the national average, in the setting of also harboring a high ARI associated mortality. This may have meant less availability, access, awareness, or cultural differences in treatment. Flu vaccinations may also be a potential option for areas with significantly high incidence of severe pneumonia such as the Mid-Western region [4].

The development and expansion of CB-IMCI has been a primary means of medical treatment and response care to acute illness in children. The program has expanded throughout the nation, and further included newborn care (jaundice, possible sepsis, hypothermia, poor weight gain, etc). The benefits of CB-IMCI is its community sensitive methods, data gathering, and integration of maternal, education, and immunization services. CB-IMCI has been transforming more than an acute care service, and continues to play a pivotal role in reducing infant and child mortality [4,10].

Considering the substantial strides that community based care has met according to data, the rise of an ideal health care system emerges. The model of community based integrated care has already shown success in closing the disparity gap, delivering acute care, outreach/education, and even primary care. Most efforts have been dealt with in a top-down fashion, meaning health care has been approaching disease entities. Furthermore, interventions have mainly been singular; i.e. the campaign for Japanese encephalitis or delivery of education on breast feeding separately. The grassroots nature of this healthcare delivery has been very beneficial in the setting of a difficult topography. There has been extensive evidence that community based care provides effective, long term, and affordable provisions for infants and children, as presented in said compiled annual data. Though there is inadequate assessment of growth monitoring, chronic malnutrition, micronutrient deficiency, region specific acute illness epidemiology and severity. According to health care deficits mentioned, alongside strengths in health policy in the past decade, it has become evident that standardized primary care for children should be established $[4,9]$.

\subsection{Limitations}

Data reporting in annual reports held a significant limitation, as data collection presented with many discrepancies and gaps. This may be explained by data not being reported in timely manner (70\% of data). It was difficult to obtain data from non-public facilities. Significant data was not included from thee non-public sectors of care and suggest some underreporting. No statistical significant was calculated with data.

Furthermore, data reported infant mortality as an average for a ten year period rather than an annual result. Child under-5 mortality was reported as an average per five years. This may suggest that the first year the actual mortality may be very different than the fifth year. This does not provide an accurate picture of timely data.

Financial and systems operations were not analyzed. Information regarding these factors highly impact public health interventions, such as immunization campaigns. Epidemiology of specific diseases, with pathological data was not analyzed, except certain vaccine preventable diseases.

\subsection{Recommendations}

1. Data collection to be reported annually rather than across 5 or 10 years. This change would yield more up to date results and allow for quicker health care policy responses

2. Further research is needed regarding mountain communities, other ecological descriptors, minor groups, by caste, etc to identify at-risk and vulnerable populations. Data can direct future interventions and resource allocation, which should be very dynamic.

3. Target Measles vaccination coverage for the Western region. Encourage follow up in the Central, Mid and Far Western regions. One possible suggestion is to pilot incentivizing immunizations. Incorporate TT vaccination in antenatal care. Allocating JE lab confirmation seasonally. All these methods can encourage immunization utilization and coverage, as well as proper resource allocation. Considering financial constraints for vaccines, resource allocation based on disease prevalence (case-based) can be more efficient when delivering JE, Measles-Rubella, or perhaps even Flu vaccinations.

4. Encourage SUN / Multi-Sector Nutrition Plan for improved food security in more western regions with interim nutrient supplementation. Addressing the underlying concern with child health care - poverty - is critical. Maintenance and strengthening of Vitamin-A, deworming, salt iodization, and iron-folate distribution programs. Collection of data regarding micronutrient deficiencies, anemia, parasite epidemiology to guide distribution of services.

5. Elaborate emergency response systems and care. Disease epidemics, natural disasters, etc have likely been causing major events of high mortality, as seen in ARI data. Sophistication of response services can appropriately and quickly respond to major disasters, supply aid, and beneficially affect recovery afterwards.

6. Improving quality care provided by CB-IMCI and current health care posts and facilities. Increasing mobile visits to communities. Continue training and advancing human resources in health care and public health. Strong recognized contributors include female community health care workers. Contributors that need work include vaccinators and mobile teams [4]. 
7. Increased utilization of the Child Health Card. Every child to have updated child health cards to standardize immunizations, trend growth curves, etc.

8. Community based primary health care. (CB-PHC). A call for an integration of all the singular services and interventions mentioned. Upscaling the CB-IMCI to a more multidisciplinary service that harnesses all the strengths of the latest CB-IMCI but incorporates preventative medicine to the current acute care model. This would include education, breast feeding, immunization delivery, nutrition and micronutrient supplementation, neonatal and well baby care (which is most crucial). Table 1 presents key features of a community based model of primary health care for children. Information from Table 1 comes from collaborate review of several global community health systems, and what makes them successful. Key features to note include community health workers and community buy-in with services. The comprehensive nature of these services were highlighted $[4,9]$.

Table 1. Key Characteristics in Long-Term Integrated Community Health Care Systems for Children [9] Common Characteristics of Eight Successful Longer-Term Integrated Programs for Improving Child Health

\begin{tabular}{|c|c|}
\hline \multicolumn{2}{|c|}{ Common Characteristics of Eight Successful Longer-Term Integrated Programs for Improving Child Health } \\
\hline Characteristics & Findings for the Eight Programs \\
\hline \multirow{3}{*}{ Range of services provided } & $\begin{array}{c}\text { Provision of a comprehensive array of preventive and curative primary health carc services } \\
\text { (child health, maternal health, reproductive health, and family planning) }\end{array}$ \\
\cline { 2 - 3 } & $\begin{array}{c}\text { Presence of a strong referral system from the community to higher levels of care at fixed } \\
\text { facilities, including hospitals with surgical capability }\end{array}$ \\
\hline \multirow{2}{*}{$\begin{array}{c}\text { Health program management nnd support } \\
\text { Nature of community } \\
\text { partncrships/community } \\
\text { involvement }\end{array}$} & $\begin{array}{c}\text { Presence of a strong system of management and supervision led by competent and dcdicatcxi } \\
\text { professionals (including maintaining needed supplies and drugs) }\end{array}$ \\
\cline { 2 - 2 } & Achievement of a record of treating patients and clients with a high level of respect \\
\hline \multirow{2}{*}{ Role of community-ba!^d workers } & $\begin{array}{c}\text { Presence of a strong partnership between the program and the community, with a strong level } \\
\text { of trust in the community toward the program }\end{array}$ \\
\cline { 2 - 2 } & $\begin{array}{c}\text { Strong training and support of community-based workers present, the workers are an integral } \\
\text { part of the program, and financial support for them is assured*1 }\end{array}$ \\
\cline { 2 - 2 } & Community-based workers achieve routine contact with all families* \\
\hline
\end{tabular}

\section{Acknowledgements}

Padmini Murthy MD, MPH, MS, MPhil, CHES, FRSPH. Asst Professor Dept of Health Policy and Management. Global Health Director NYMC. NGO Rep MWIA to the United Nations. NGOCSW NY to the United Nations Executive Committee member Chair COWR, APHA. Program Chair International Health Section APHA.

Dr Asis De. Professor, Department of Community Medicine. Manipal College of Medical Sciences, Pokhara, Nepal.

\section{References}

[1] Christian, P., Stewart, C.P., LeClerq, S.C., Wu, L., Katz, J., West Jr., K.P., and Khatry, S.K. July 2009. Antenatal and Postnatal Iron Supplementation and Childhood Mortality in Rural Nepal: A Prospective Follow-up in a Randomized, Controlled Community Trial. American Journal of Epidemiology. Baltimore, Maryland. USA. 170: 1127-1136.

[2] Department of Health Services (DoHS). 2007. Annual Report 2063/2064. Government of Nepal Ministry of Health and Population. Kathmandu, Nepal.

[3] Department of Health Services (DoHS). 2010. Annual Report 2066/2067. Government of Nepal Ministry of Health and Population. Kathmandu, Nepal.

[4] Department of Health Services (DoHS). 2012. Annual Report 2068/2069. Government of Nepal Ministry of Health and Population. Kathmandu, Nepal.

[5] Ministry of Health. Family Health Division (MoH:FHD). April 2002. Demographic Health Survey 2001. New ERA, Kathmandu, Nepal and ORC Macro, Calverton, Maryland, USA.

[6] Ministry of Health and Population. Population Division (MoHP:PD). May 2007. Demographic Health Survey 2006. New
ERA, Kathmandu, Nepal and ORC International Inc., Calverton, Maryland, USA.

[7] Ministry of Health and Population. Population Division (MoHP:PD). March 2012. Demographic Health Survey 2011. New Era, Kathmandu, Nepal and ICF International, Calverton, Maryland, USA.

[8] Pambos, M., Ng, J., Loukes, J., Matheson, J., Aryal, B., Adhikari, S., Kerry, S., Reid, F., \& Oakeshott, P. 2012. Demographics and diagnoses at rural health camps in Nepal: cross-sectional study. Family Practice 2012. Oxford University Press. 29: 528-533.

[9] Perry, H., Freeman, P., Gupta, S., \& Rassekh, B.M. July 2009. "How Effective is Community-Based Primary Health Care in Improving the Health of Children?”. Community-Based Primary Health Care Working Group, International Health Section. American Public Health Association. Available:http://www.amoshealth.org/wpcontent/uploads/2011/12/Review-of-Evidence-on-Effectivenessof-CBPHC-July-2009.pdf.[Accessed April 10 $\left.{ }^{\text {th }}, 2014\right]$.

[10] Pradhan, A., Aryal, R.H., Regmi, G., Ban, B., \& Govindasamy, P. 1997. "Nepal Family Health Survey 1996”. Ministry of Health (Nepal), New ERA, and Macro International Inc. Kathmandu, Nepal and Celverton, Maryland.

[11] Sharma, S. September 2011. “Nepal's Successes on Micronutrients and Progress on Multi-sectoral Nutrition Planning with a Vision to Decentralize Scale-Up of Nutrition”. Ministry of Health and Population and UNICEF. Nepal and New York, USA. Available: http://scalingupnutrition.org/wpcontent/uploads/2012/09/archived/Nepal-SUN-NYC-EE-011.pdf. [Accessed April 10 ${ }^{\text {th }}, 2014$ ].

[12] Shrestha, I.B. \& Pathak, L.R. Aug 2012. "Review of the National Health Policy 1991”. National Health Sector Support Program. Available:

http://www.nhssp.org.np/health_policy/Review\%20of\%20Nationa 1\%20Health\%20Policy\%201991.pdf. [Accessed March 28, 2014].

[13] UK Department for International Development (DFID). May, 2013. Regional Dimensions of Poverty and Vulnerability in Nepal: Summary Report. UKAID, UK. Available: https://www.gov.uk/government/uploads/system/uploads/attachme nt_data/file/204015/Regional-Dimensions-Poverty-Nepal.pdf. [Accessed April 10, 2014].

[14] World Health Organization. 2011. Nepal: Health Profile. Available: http://www.who.int/gho/countries/npl.pdf. [Accessed January $\left.22^{\text {nd }}, 2014\right]$. 